\title{
Adaptive Surrogate Model with Active Refinement Combining Kriging and a Trust Region Method
}

\author{
B. Gaspar \\ Centre for Marine Technology and Ocean Engineering (CENTEC) \\ Instituto Superior Técnico, Universidade de Lisboa, Portugal
}

A.P. Teixeira

Centre for Marine Technology and Ocean Engineering (CENTEC)

Instituto Superior Técnico, Universidade de Lisboa, Portugal

C. Guedes Soares

Centre for Marine Technology and Ocean Engineering (CENTEC)

Instituto Superior Técnico, Universidade de Lisboa, Portugal

\begin{abstract}
In the present paper an adaptive Kriging surrogate model with active refinement is proposed to solve component reliability analysis problems (i.e. with a single design point) with a reasonable limit for the dimensionality of the basic random variables space. The model uses an adaptive Kriging-based trust region method to search for the design point and predict the failure probability based on the first-order reliability method. This prediction is then verified or improved using Monte Carlo simulation with importance sampling based on a Kriging surrogate model built up iteratively around the design point using an active refinement algorithm. The usefulness of the proposed surrogate model in terms of accuracy and efficiency for practical engineering applications is shown with a numerical example involving an advanced nonlinear FEA structural model.
\end{abstract}

\section{INTRODUCTION}

The use of advanced numerical models (e.g. finite element analysis (FEA) structural models) in the context of structural reliability analysis imposes some challenges due to the large computational cost that may be involved. The strategy that has been adopted to alleviate this problem is to replace the time-consuming numerical model by a so-called surrogate model (also known as meta-model or response surface model), which can be evaluated at rather low computational cost (e.g. Bucher and Most 2008, Sudret 2012).

Examples of surrogate models that have been used in this context are polynomial regression models (e.g. Bucher and Bourgund 1990), artificial neural networks (e.g. Hurtado and Alvarez 2001), support vector machines (e.g. Bourinet et al. 2011) and Kriging models also known as Gaussian process models (e.g. Romero et al. 2004, Kaymaz 2005, Bichon et al. 2008, Echard et al. 2011, Schöbi and Sudret 2014).

This strategy has been the most used and promising one for practical engineering applications. However, alternative approaches based on stochastic finite element methods and direct evaluation of the numerical model can also be considered in some problems (e.g. Matthies et al. 1997, Teixeira and Guedes Soares 2007).

Well-established and efficient methods to compute small failure probabilities such as the first- and second-order reliability methods (FORM/SORM) or Monte Carlo simulation (MCS) methods with variance reduction techniques (e.g. Bucher 2009) can then be used at low computational cost. An accurate surrogate model approximation in the region of the basic random variables space that most contributes to the failure probability integral is however 
required (e.g. Bucher and Bourgund 1990, Bucher and Most 2008).

To satisfy the latter requirement efficiently, several adaptive algorithms have been developed in the last years since the first proposal by Bucher and Bourgund (1990). The use of Kriging models in this context have been explored by several authors (e.g. Kaymaz 2005, Bichon et al. 2008, Echard et al. 2011, Echard et al. 2013, Schöbi and Sudret 2014), due to their interesting features for structural reliability analysis as the interpolation capability, large flexibility and prediction uncertainty measure (e.g. Santner et al. 2003, Gaspar et al. 2014a).

In the present paper an adaptive Kriging surrogate model with active refinement is proposed for structural reliability analysis with nonlinear and time-consuming implicit limit state functions (e.g. requiring the evaluation of a FEA model). The proposed model uses the adaptive Kriging-based trust region method proposed by Gaspar et al. (2014b) to search for the design point, which combines the adaptive trust region algorithm proposed by Zou et al. (2002) and Stocki et al. (2009) with Kriging models.

The design point found provides a failure probability prediction based on FORM. This prediction is then verified or improved using MCS with importance sampling (MCS-IS) based on a Kriging model built up iteratively around the FORM design point using the active refinement algorithm proposed by Echard et al. (2013). The accuracy and efficiency of the proposed surrogate model is shown in the paper with a numerical example.

\section{KRIGING SURROGATE MODELS}

The Kriging models are exact interpolation models based on the idealization of the numerical model response as the realization of a Gaussian process (e.g. Santner et al. 2003, Echard et al. 2011). In the context of structural reliability analysis these models can be used to approximate a time-consuming implicit limit state function as:
$G(\boldsymbol{x})=\boldsymbol{f}(\boldsymbol{x})^{\top} \boldsymbol{\beta}+Z(\boldsymbol{x})$

where $\boldsymbol{f}(\boldsymbol{x})^{\top} \boldsymbol{\beta}=\beta_{1} f_{1}(\boldsymbol{x})+\ldots+\beta_{p} f_{p}(\boldsymbol{x})$ is a linear regression model that defines the mean or trend of the Kriging model and $Z(\boldsymbol{x})$ is a zero mean and stationary Gaussian process.

A zero-order polynomial (constant) is in general adopted as regression model, defining a so-called ordinary Kriging model (e.g. Echard et al. 2011). The use of higher-order polynomials does not provide in general significant gains of accuracy (Gaspar et al. 2014a). The stationary Gaussian process has covariance defined by:

$C_{Z Z}\left(\boldsymbol{x}, \boldsymbol{x}^{\prime}\right)=\sigma_{Z}^{2} \mathcal{R}\left(\boldsymbol{\theta} ; \boldsymbol{x}, \boldsymbol{x}^{\prime}\right)$

with $\sigma_{Z}^{2}$ the variance and $\mathcal{R}$ the autocorrelation function. A square exponential (Gaussian) autocorrelation function of the form:

$\mathcal{R}\left(\boldsymbol{\theta} ; \boldsymbol{x}, \boldsymbol{x}^{\prime}\right)=\prod_{i=1}^{n} \exp \left(-\theta_{i}\left(x_{i}-x_{i}^{\prime}\right)^{2}\right)$

can be used for structural reliability analysis, with $\theta_{i}(i=1, \ldots, n)$ correlation parameters (e.g. Kaymaz 2005, Echard et al. 2011).

The determination of the Kriging model parameters requires a model fitting technique and a sample of support points:

$\boldsymbol{X}^{(k)}=\left\{\boldsymbol{x}^{(k)}, y^{(k)}=G\left(\boldsymbol{x}^{(k)}\right)\right\}, \quad k=1, \cdots, m$

with realizations of the vector of basic random variables $\boldsymbol{x}=\left[x_{1}, \ldots, x_{n}\right]^{\top}$ and the corresponding values of the true limit state functions $y=G(\boldsymbol{x})$. The correlation parameters can be determined through maximum likelihood estimation, with a generalized least squares solution for the regression coefficients and process variance (e.g. Santner et al. 2003, Echard et al. 2011). The optimal set of parameters $\boldsymbol{\theta}^{*} \equiv \widehat{\boldsymbol{\theta}}, \boldsymbol{\beta}^{*} \equiv \widehat{\boldsymbol{\beta}}$ and $\sigma_{Z}^{2 *} \equiv \hat{\sigma}_{Z}^{2}$ is then defined.

The prediction of a Kriging model is by definition a Gaussian random variable with mean value and variance given by:

$\mu_{\hat{G}}(\boldsymbol{x})=\boldsymbol{f}(\boldsymbol{x})^{\top} \hat{\boldsymbol{\beta}}+\boldsymbol{r}(\boldsymbol{x})^{\top} \boldsymbol{R}^{-1}(\boldsymbol{y}-\boldsymbol{F} \hat{\boldsymbol{\beta}})$ 


$$
\begin{gathered}
\sigma_{\hat{G}}^{2}(\boldsymbol{x})=\hat{\sigma}_{Z}^{2}\left[1+\boldsymbol{u}(\boldsymbol{x})^{\top}\left(\boldsymbol{F}^{\top} \boldsymbol{R}^{-1} \boldsymbol{F}\right)^{-1} \boldsymbol{u}(\boldsymbol{x})-\right. \\
\left.\boldsymbol{r}(\boldsymbol{x})^{\top} \boldsymbol{R}^{-1} \boldsymbol{r}(\boldsymbol{x})\right]
\end{gathered}
$$

with $\boldsymbol{u}(\boldsymbol{x})=\boldsymbol{F}^{\top} \boldsymbol{R}^{-1} \boldsymbol{r}(\boldsymbol{x})-\boldsymbol{f}(\boldsymbol{x})$. The vector $\boldsymbol{r}(\boldsymbol{x})$ defines the correlations between the prediction point $\boldsymbol{x}$ and the sample of points $\boldsymbol{x}^{(k)}(i=1, \ldots, m)$ in Eq. (4), the matrix $\boldsymbol{R}$ defines the correlation between each pair of points $\boldsymbol{x}^{(k)}(i=1, \ldots, m)$ and $\boldsymbol{F}$ is the regression matrix (e.g. Santner et al. 2003, Echard et al. 2011).

The mean value in Eq. (5) defines the surrogate model prediction and the variance in Eq. (6) defines the prediction uncertainty (i.e. epistemic uncertainty associated with the finite number of support points). These two equations satisfy $\left\{\mu_{\hat{G}}\left(\boldsymbol{x}^{(k)}\right)=G\left(\boldsymbol{x}^{(k)}\right), \hat{\sigma}_{\hat{G}}^{2}\left(\boldsymbol{x}^{(k)}\right)=0\right\}(k=$ $1, \ldots, m)$, which means that the Kriging model is an exact interpolator.

\section{ADAPTIVE KRIGING SURROGATE MODEL WITH ACTIVE REFINEMENT}

The proposed adaptive surrogate model is formulated in the standard normal space of basic random variables $\boldsymbol{u}$ (i.e. uncorrelated normal variables with zero mean and unit variance). In this space the adaptive surrogate model involves two main steps based on the initial proposals of Zou et al. (2002) and Echard et al. (2013).

The first step consists in the design point search and failure probability prediction based on FORM. The adaptive Kriging-based trust region method proposed by Gaspar et al. (2014b) is used for this purpose. This method is based on the search algorithm proposed by Zou et al. (2002) and Stocki et al. (2009). This approach differs from the proposal of Echard et al. (2013), which uses FORM with direct evaluation of the true implicit limit state function.

In the second step the failure probability prediction based on FORM is improved using AK-MCS-IS at the approximate design point found in the first step, as proposed by Echard et al. (2013). The importance of this improvement increases with the nonlinearity of the true limit state function around the design point, which in practice is not known in advance.

\subsection{Design point search and failure probability prediction based on FORM}

The adaptive Kriging-based trust region method proposed by Gaspar et al. (2014b) uses the Kriging mean prediction in Eq. (5) as surrogate model for the true implicit limit state function. The surrogate model is defined in successive trust regions (i.e. subsets $S$ of the standard normal space) which the location and size are updated adaptively until the true design point is found within a prescribed accuracy.

Each trust region is defined by a hypercube with center point $c=\left[c_{1}, \ldots, c_{n}\right]^{\top}$ and side length $s$. The corresponding subset $S$ is defined by the intervals $u_{i} \in\left[c_{i}-s / 2, c_{i}+s / 2\right](i=1, \ldots, n)$. The notation $S=\{c, s\}$ is used to define a trust region with center point $c$ and side length $s$. The adaptive method consists in successive iterations of the location and size of the trust region $S$ based on the following algorithm:

Step 1: Define an initial trust region $S^{(i)}=\left\{\boldsymbol{c}^{(i)}\right.$, $\left.s^{(i)}\right\}(i=1)$. The initial center point can be located at the origin of the standard normal space and the side length should be large enough to cross the true implicit limit state surface $G(\boldsymbol{u})=0$ (i.e. including a region of the safe domain $\mathcal{D}_{s}$ and a region of the failure domain $\mathcal{D}_{f}$ ). Without prior knowledge of the problem $s^{(1)}=6$ can be used as initial trust region size.

Step 2: Generate a sample $\boldsymbol{X}^{(k)}(k=1, \ldots, m)$ of support points inside the current trust region $S^{(i)}$. The support points are defined by realizations $\boldsymbol{u}^{(k)}(k=1, \ldots, m)$ uniformly distributed. A Latin hypercube sampling technique with midpoint location and spurious correlation reduction is used for this purpose (Olsson et al. 2003). All support points are stored in a database of support points.

Step 3: Define a Kriging surrogate model for the true implicit limit state function in the current 
trust region $S^{(i)}$. This step consists in fitting a Kriging model. All support points in the database of support points that are inside the current trust region are used in the model fitting.

Step 4: Find a local design point $\boldsymbol{u}^{*(i)}$ for the current trust region $S^{(i)}$ by solving the following constrained optimization problem based on the FORM algorithm:

$\boldsymbol{u}^{*(i)}=\underset{\boldsymbol{u}}{\arg \min }\left\{\|\boldsymbol{u}\| \mid \mu_{\hat{G}}^{(i)}(\boldsymbol{u}) \leq 0\right\}$.

The local design point found defines an additional support point. The trust region center point can be used as starting point.

Step 5: Update the trust region $S^{(i)}(i=i+1)$. The updated center point and side length are defined based on the location of the local design point $\boldsymbol{u}^{*(i-1)}$ found in Step 4 according to the conditions: 1) $\boldsymbol{c}^{(i)}=\boldsymbol{u}^{*(i-1)}$ and $s^{(i)}=s^{(i-1)} / 2$, if the local design point $\boldsymbol{u}^{*(i-1)}$ is located inside or on the boundary of the previous trust region $S^{(i-1)}$; 2) $\boldsymbol{c}^{(i)}=\mathcal{P}\left(\boldsymbol{u}^{*(i-1)}\right)$ and $s^{(i)}=s^{(i-1)}$, otherwise. $\mathcal{P}$ is an operator that defines the coordinates of the projection of $\boldsymbol{u}^{*(i-1)}$ on the boundary of the trust region $S^{(i-1)}$ in the direction of its center point.

Step 6: Perform successive iterations of the trust region $S^{(i)}=\left\{c^{(i)}, s^{(i)}\right\}$ based on Steps 2 to 5 until the following convergence criteria are satisfied:

$$
\frac{\left\|\boldsymbol{c}^{(i)}-\boldsymbol{c}^{(i-1)}\right\|}{\left\|\boldsymbol{c}^{(i-1)}\right\|} \leq \varepsilon_{c} \quad \text { and } \quad\left|G\left(\boldsymbol{u}^{*(i-1)}\right)\right| \leq \varepsilon_{g}\left|G_{o}\right|
$$

with $G_{o}$ the true implicit limit state function value at the origin of the standard normal space, $\varepsilon_{c}$ a prescribed error limit (e.g. $\varepsilon_{c}=0.10$ ) for the trust region center point and $\varepsilon_{g}$ a prescribed error limit (e.g. $\varepsilon_{g}=0.01$ ) for the true implicit limit state function at the approximate design point found in Step 4.

The convergence of the adaptive method is only considered if the criteria in Eq. (8) are satisfied with the approximate design point found in Step 4 located inside or on the boundary of the trust region. The total number of evaluations of the true implicit limit state function required by the adaptive method is $N_{g}=1+N_{r}(m+1)$, where $m$ is the number of support points generated in Step 2 per trust region iteration and $N_{r}$ is the total number of iterations.

After convergence of the adaptive method a failure probability prediction based on FORM is readily available. The design point found in Step 4 in the last trust region iteration defines the Hasofer-Lind reliability index $\beta=\left\|\boldsymbol{u}^{*}\right\|$ and the FORM failure probability $p_{f} \approx \Phi(-\beta)$, with $\Phi$ the standard normal probability distribution function.

\subsection{Failure probability prediction based on $A K$ - MCS-IS}

An unbiased estimator for the failure probability prediction based on MCS-IS can be given by (e.g. Bucher 2009):

$\hat{p}_{f}=\frac{1}{m_{I S}} \sum_{i=1}^{m_{I S}} I_{f}\left(\boldsymbol{u}^{(i)}\right) \frac{\phi_{n}\left(\boldsymbol{u}^{(i)}\right)}{\varphi_{n}\left(\boldsymbol{u}^{(i)}\right)}$

with $\phi_{n}(\boldsymbol{u})$ the standard normal probability density function and $\varphi_{n}(\boldsymbol{u})$ the importance sampling probability density function (i.e. $n$ dimensional normal with mean $\boldsymbol{\mu}=\boldsymbol{u}^{*}$ and covariance $\boldsymbol{C}_{U U}=\boldsymbol{I}$ ).

$\mathcal{I}_{f}$ is an indicator function for the failure domain defined as: $I\left(\boldsymbol{u}^{(i)}\right)=1$ if $\mu_{\hat{G}}\left(\boldsymbol{u}^{(i)}\right) \leq 0$ and $I\left(\boldsymbol{u}^{(i)}\right)=0$ if $\mu_{\widehat{G}}\left(\boldsymbol{u}^{(i)}\right)>0$, with $\mu_{\widehat{G}}(\boldsymbol{u})$ the Kriging prediction mean defined by Eq. (5) and $\boldsymbol{u}^{(i)}\left(i=1, \ldots, m_{I S}\right)$ realizations of $\varphi_{n}(\boldsymbol{u})$. It should be mentioned that $\mu_{\widehat{G}}(\boldsymbol{u})=0$ is used as surrogate model for the true implicit limit state surface $G(\boldsymbol{u})=0$ in the underlying MCS-IS classification problem.

The statistical uncertainty associated with the failure probability prediction in Eq. (9) can be quantified by the prediction variance:

$\sigma_{\hat{p}_{f}}^{2}=\frac{\frac{1}{m_{I S}} \sum_{i=1}^{m_{I S}} I_{f}\left(\boldsymbol{u}^{(i)}\right) \frac{\phi_{n}\left(\boldsymbol{u}^{(i)}\right)^{2}}{\varphi_{n}\left(\boldsymbol{u}^{(i)}\right)^{2}}-\hat{p}_{f}^{2}}{m_{I S}-1}$ 
or alternatively by the corresponding coefficient of variation $\operatorname{Cov}\left[\hat{p}_{f}\right]=\sigma_{\hat{p}_{f}} / \hat{p}_{f}$.

The active refinement algorithm proposed by Echard et al. (2013) is used to refine iteratively the Kriging surrogate model used in the MCS-IS failure probability prediction. The algorithm is briefly described next and a more detailed description can be found in the original paper (Echard et al. 2013).

The support points in the database that are located inside a trust region with center point $\boldsymbol{c}=\boldsymbol{u}^{*}$ and side length $s=6$ are used as initial support points to start the refinement algorithm. This sample of support points is denoted as $\boldsymbol{X}_{o}^{(k)}$ $\left(k=1, \ldots, m_{A R}\right)$. The support points outside this trust region are not considered as they are located in regions of the basic random variables space with small $\varphi_{n}(\boldsymbol{u})$ values.

A sample $\boldsymbol{u}^{(i)}\left(i=1, \ldots, m_{I S}\right)$ of realizations of $\varphi_{n}(\boldsymbol{u})$ is generated and the Kriging model (i.e. prediction mean $\mu_{\widehat{G}}(\boldsymbol{u})$ and variance $\left.\sigma_{\hat{G}}^{2}(\boldsymbol{u})\right)$ is evaluated at this sample. A failure probability prediction based on MCS-IS can then be computed using Eq. (9), with its statistical uncertainty quantified by $\operatorname{Cov}\left[\hat{p}_{f}\right]$.

The learning function proposed by Echard et al. (2011),

$U(\boldsymbol{u})=\frac{\left|\mu_{\hat{G}}(\boldsymbol{u})\right|}{\sigma_{\hat{G}}(\boldsymbol{u})}$

is used in the active refinement algorithm to identify the best realization of $\varphi_{n}(\boldsymbol{u})$ in the sample $\boldsymbol{u}^{(i)}\left(i=1, \ldots, m_{I S}\right)$ to be added to the sample of support points $\boldsymbol{X}_{o}^{(k)}\left(k=1, \ldots, m_{A R}\right)$. The aim is to increase the accuracy of the limit state surface $\mu_{\widehat{G}}(\boldsymbol{u})=0$ predicted by the Kriging model and consequently increase the accuracy of the MCS-IS failure probability prediction.

This learning function favors realizations of $\varphi_{n}(\boldsymbol{u})$ that can be misclassified according to the sign of the Kriging model prediction mean $\mu_{\widehat{G}}(\boldsymbol{u})$. These misclassifications typically occur in points located in the vicinity of the limit state surface $\left(\mu_{\widehat{G}}(\boldsymbol{u}) \approx 0\right)$, in points with a large prediction uncertainty $\left(\sigma_{\widehat{G}}(\boldsymbol{u}) \gg 0\right)$ or in points where both situations occur (Echard et al. 2011).

The realization of $\varphi_{n}(\boldsymbol{u})$ in the sample $\boldsymbol{u}^{(i)}\left(i=1, \ldots, m_{I S}\right)$ that minimizes the learning function $U(\boldsymbol{u})$ in Eq. (11),

$$
\boldsymbol{u}_{o}=\underset{\boldsymbol{u}^{(i)}\left(i=1, \cdots, m_{I S}\right)}{\arg \min }\{U(\boldsymbol{u})\}
$$

is selected to define the next support point to be added to the sample $\boldsymbol{X}_{o}^{(k)}\left(k=1, \ldots, m_{A R}\right)$. This additional support point requires one evaluation of the true implicit limit state function $G(\boldsymbol{u})$. A new Kriging model is then defined based on this refined sample of support points and a new MCS-IS failure probability prediction is computed.

The active refinement algorithm proceeds until the stopping condition $U\left(\boldsymbol{u}_{o}\right) \geq 2$ is satisfied, with $\boldsymbol{u}_{o}$ given by Eq. (12) (Echard et al. 2011). This condition ensures that the Kriging model prediction mean $\mu_{\widehat{G}}(\boldsymbol{u})$ is at least twice the prediction standard deviation $\sigma_{\hat{G}}(\boldsymbol{u})$ at each realization $\boldsymbol{u}^{(i)}\left(i=1, \ldots, m_{I S}\right)$. The corresponding probability of correct classification (i.e. failure or safe domain with the function $I_{f}$ in Eq. (9)) is $\Phi(2)=0.977$.

\section{NUMERICAL EXAMPLE}

The numerical example involves the reliability assessment of a stiffened plate element under uniaxial compression typical of the deck structure of double hull oil tanker ships (Figure 1). This numerical example was used in previous studies by Gaspar et al. (2014a,c), where a detailed description is given. It is representative of a possible practical engineering application of the proposed adaptive surrogate model as it requires a time-consuming nonlinear FEA.

The limit state function associated with this numerical example is given by:

$G(\boldsymbol{x})=\sigma_{x u}-\left(\sigma_{x, s w}+\sigma_{x, w v}\right)$

with $\sigma_{x u}(\boldsymbol{x})=\max _{\varepsilon_{x}}\left\{\sigma_{x}\left(\boldsymbol{x} ; \varepsilon_{x}\right)\right\}$ the ultimate compressive stress of the stiffened plate element and $\sigma_{x, s w}$ and $\sigma_{x, w v}$ the still water and wave- 
induced components of the uniaxial compressive stress induced by the ship hull girder vertical bending moments (e.g. Gaspar et al. 2011).

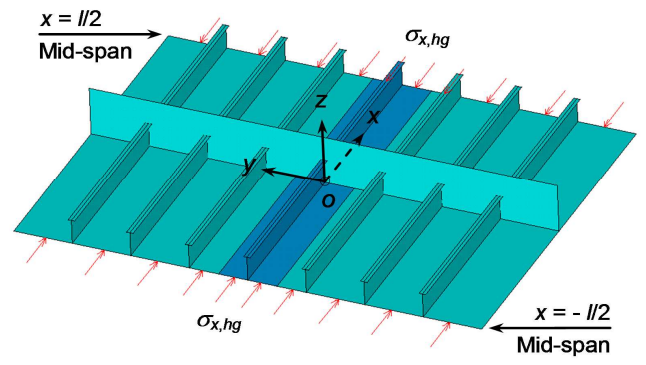

Figure 1: Stiffened plate under uniaxial compression implemented in the FEA code ANSYS (Paik et al. 2008, Gaspar et al. 2014c).

The basic random variables and the corresponding probabilistic models involved in Eq. (13) are given in Table 1. The following notation is adopted: $t$ plate thickness; $t_{w}$ stiffener web thickness; $t_{f}$ stiffener flange thickness; $\sigma_{y d, p}$ plate material yield stress; $\sigma_{y d, s}$ stiffener material yield stress; $E_{p}$ plate material Young's modulus; $E_{s}$ stiffener material Young's modulus; $w_{o p}$ plate initial distortion amplitude; $w_{o s}$ stiffener sideways initial distortion amplitude; $w_{o c}$ stiffener column-type initial distortion amplitude. All variables are considered to be uncorrelated.

Table 1: Random variables and probabilistic models.

\begin{tabular}{ccccc}
\hline Variable & Units & Mean & Cov & Prob. Dist. \\
\hline$t$ & $\mathrm{~mm}$ & 20.5 & 0.02 & Normal \\
$t_{w}$ & $\mathrm{~mm}$ & 9.5 & 0.02 & Normal \\
$t_{f}$ & $\mathrm{~mm}$ & 14.0 & 0.02 & Normal \\
$\sigma_{y d, p}$ & $\mathrm{MPa}$ & 348.3 & 0.06 & Lognormal \\
$\sigma_{y d, s}$ & $\mathrm{MPa}$ & 348.3 & 0.06 & Lognormal \\
$E_{p}$ & $\mathrm{MPa}$ & 206000 & 0.06 & Lognormal \\
$E_{s}$ & $\mathrm{MPa}$ & 206000 & 0.06 & Lognormal \\
$w_{o p}$ & $\mathrm{~mm}$ & 2.3 & 0.50 & Lognormal \\
$w_{o s}$ & $\mathrm{~mm}$ & 2.8 & 0.50 & Lognormal \\
$w_{o c}$ & $\mathrm{~mm}$ & 2.8 & 0.50 & Lognormal \\
$\sigma_{x, s w}$ & $\mathrm{MPa}$ & 39.8 & 0.29 & Normal \\
$\sigma_{x, w v}$ & $\mathrm{MPa}$ & 123.4 & 0.09 & Gumbel \\
\hline
\end{tabular}

Table 2: True failure probability predictions.

\begin{tabular}{ccccc}
\hline Method & $p_{f}$ & $\operatorname{Cov}\left[p_{f}\right]$ & $\beta$ & $N_{g}$ \\
\hline FORM & $9.709 \cdot 10^{-6}$ & - & 4.271 & 126 \\
MCS-IS & $1.901 \cdot 10^{-5}$ & 0.015 & 4.119 & $126+5.0 \cdot 10^{4}$ \\
\hline * Convergence criteria: $\varepsilon_{u}{ }^{*}=0.001$ and $\varepsilon_{g}=0.001$
\end{tabular}

The true failure probability predictions based on FORM and MCS-IS are given in Table 2. The MCS-IS prediction was defined by the mean value of 100 runs in order to eliminate some possible bias due to the underlying statistical uncertainty of the method. A Kriging Sampling Model (KSM) was used as surrogate for $\sigma_{x u}$ in Eq. (13). A detailed description of this approach can be found in Gaspar et al. (2014a).

The failure probabilities predicted by the adaptive surrogate model are given in Table 3 and Table 4 in terms of statistics for 100 runs. The relative error $\delta_{p}=\left(\hat{p}_{f}-p_{f}\right) / p_{f}$ is used as accuracy measure, with $\hat{p}_{f}$ the predicted value and $p_{f}$ the true value in Table 2. $m=50$ support points per trust region iteration were adopted in Step 1. The trust region convergence criteria were set to $\varepsilon_{c}=0.10$ and $\varepsilon_{g}=0.01$. The AKMCS-IS prediction in Step 2 is based on $m_{I S}=1.0 \cdot 10^{4}$ realizations of $\varphi_{n}(\boldsymbol{u})$.

Table 3: Failure probability prediction based on FORM in Step 1 of the adaptive surrogate model.

\begin{tabular}{cccccc}
\hline Statistic & $\hat{p}_{f}\left(10^{-6}\right)$ & $\hat{\beta}$ & $\delta_{p}(\%)$ & $N_{r}$ & $N_{g}$ \\
\hline Mean & 9.713 & 4.271 & 0.05 & 5 & 241 \\
$C I_{0.95}^{-}$ & 9.680 & 4.271 & -0.30 & 4 & 190 \\
$C I_{0.95}^{+}$ & 9.746 & 4.272 & 0.39 & 6 & 291 \\
\hline
\end{tabular}

Table 4: Failure probability prediction based on AKMCS-IS in Step 2 of the adaptive surrogate model.

\begin{tabular}{|c|c|c|c|c|c|c|}
\hline \multicolumn{3}{|c|}{ Statistic $\hat{p}_{f}\left(10^{-5}\right) \operatorname{Cov}\left[\hat{p}_{f}\right]$} & $\hat{\beta}$ & $\delta_{p}(\%)$ & $N_{a}$ & $N_{g} *$ \\
\hline \multicolumn{7}{|c|}{ Active refinement start } \\
\hline Mean & 2.390 & 0.037 & 4.066 & 25.8 & 0 & 241 \\
\hline$C I_{0.95}^{-}$ & 2.144 & - & 4.043 & 12.8 & 0 & 190 \\
\hline$C I_{0.95}^{+}$ & 2.637 & - & 4.090 & 38.7 & 0 & 291 \\
\hline \multicolumn{7}{|c|}{ Active refinement end } \\
\hline Mean & 1.889 & 0.031 & 4.121 & -0.64 & 551 & 792 \\
\hline$C I_{0.95}^{-}$ & 1.766 & - & 4.106 & -7.11 & 506 & 719 \\
\hline$C I_{0.95}^{+}$ & 2.012 & - & 4.136 & 5.83 & 595 & 864 \\
\hline
\end{tabular}

*Accumulated $G$ evaluations: Step $1+$ Step 2

The mean relative error associated with the true failure probability prediction based on FORM is $\delta_{p}=0.05 \%$ with $N_{g}=241$ evaluations of the true limit state function. It should be mentioned that $N_{g}$ can be reduced by considering a smaller number $m$ of support points, however 
at the cost of penalizing the accuracy of the FORM prediction. The true failure probability based on MCS-IS can be predicted with a mean relative error of $\delta_{p}=-0.64 \%$, however at the cost of $N_{a}=551$ additional evaluations of the true limit state function (i.e. additional support points in the Kriging model). The statistical uncertainty of this prediction is $\operatorname{Cov}\left[\hat{p}_{f}\right]=0.031$.

The active refinement algorithm is determinant for the accuracy achieved in the MCS-IS prediction, as can be concluded from the difference in $\delta_{p}$ at the start and end iterations of the algorithm (Table 4 and Figure 2). However, the stopping condition adopted can be considered too conservative as $\delta_{p}$ tends to stabilize several iterations before $U\left(\boldsymbol{u}_{o}\right) \geq 2$ is satisfied (Figure 2) (Schöbi and Sudret 2014).

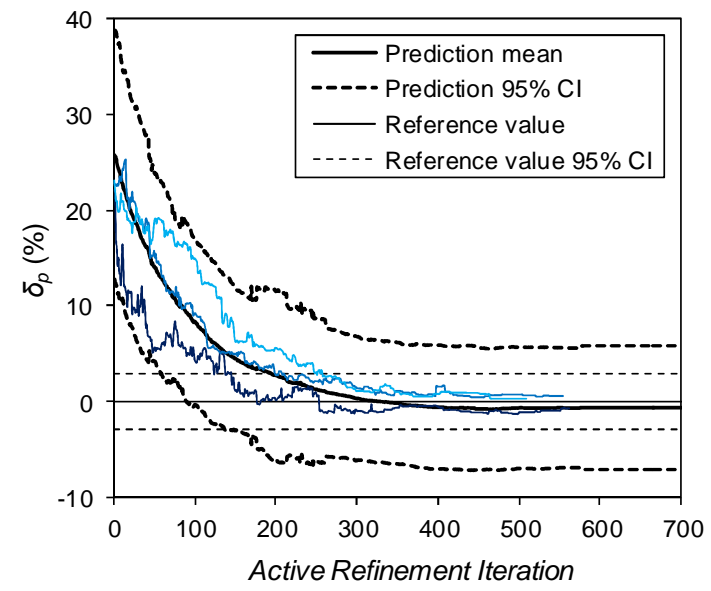

Figure 2: Mean relative error and $95 \%$ confidence interval for the AK-MCS-IS prediction in Step 2 of the adaptive surrogate model as a function of the active refinement iteration.

Figure 2 shows that approximately $N_{a}=250$ additional support points are sufficient to provide a mean relative error $\delta_{p}$ in the MCS-IS prediction of magnitude comparable to the error associated with the statistical uncertainty of the reference failure probability in Table 2 .

\section{CONCLUDING REMARKS}

An adaptive Kriging surrogate model with active refinement was proposed for structural reliability analysis with nonlinear and time-consuming implicit limit state functions. The model can in principle be used to solve any component reliability analysis problem (i.e. with a single design point), with a reasonable limit for the dimensionality of the random variables space.

The proposed adaptive surrogate model has shown to provide accurate failure probability predictions based on FORM and MCS-IS with a moderate computational cost. The active refinement algorithm implemented has shown to be determinant for an efficient control of the accuracy of the MCS-IS predictions.

Although a high level of accuracy can be achieved in the MCS-IS predictions (i.e. errors of magnitude comparable to the error associated with the statistical uncertainty of the method), the stopping condition adopted in the active refinement algorithm has shown to be too conservative. Alternative stopping conditions should therefore be investigated in future studies.

\section{ACKNOWLEDGEMENTS}

The first author has been funded by the Portuguese Foundation for Science and Technology (Fundação para a Ciência e a Tecnologia-FCT) under the contract SFRH/BPD/85093/2012. This work contributes to the project "Adaptive Methods for Reliability Analysis of Complex Structures", which is funded by the Portuguese Foundation for Science and Technology (Fundação para a Ciência e a Tecnologia-FCT) under the contract PTDC/ECM/115932/2009.

\section{REFERENCES}

Bichon, B.J., Eldred, M.S., Swiler, L.P., Mahadevan, S. \& McFarland, J.M. (2008). Efficient Global Reliability Analysis for Nonlinear Implicit Performance Functions. AIAA Journal 46(10), 2459-68.

Bourinet, J.-M., Deheeger, F. \& Lemaire, M. (2011). Assessing Small Failure Probabilities by Combined Subset Simulation and Support Vector Machines. Structural Safety 33(6), 34353.

Bucher, C. (2009). Computational Analysis of Randomness in Structural Mechanics. Taylor $\&$ Francis Group, London. 
Bucher, C.G. \& Bourgund, U. (1990). A Fast and Efficient Response Surface Approach for Structural Reliability Problems. Structural Safety 7(1), 57-66.

Bucher, C. \& Most, T. (2008). A Comparison of Approximate Response Functions in Structural Reliability Analysis. Probabilistic Engineering Mechanics 23(2-3), 154-63.

Echard, B., Gayton, N. \& Lemaire, M. (2011). AKMCS: An Active Learning Reliability Method Combining Kriging and Monte Carlo Simulation. Structural Safety 33(2), 145-54.

Echard, B., Gayton, N., Lemaire, M. \& Relun, N. (2013). A Combined Importance Sampling and Kriging Reliability Method for Small Failure Probabilities with Time-demanding Numerical Models. Reliability Engineering and System Safety 111, 232-240.

Gaspar, B., Teixeira, A.P. \& Guedes Soares, C. (2014a). Assessment of the Efficiency of Kriging Surrogate Models for Structural Reliability Analysis. Probabilistic Engineering Mechanics 37, 24-34.

Gaspar, B., Teixeira, A.P. \& Guedes Soares, C. (2014b). Structural Reliability Analysis Combining Kriging Surrogate Models with an Adaptive Trust Region Method. In Proceedings of the 7th International Conference on Computational Stochastic Mechanics (CSM-7), Deodatis, G. \& Spanos, P.D. (eds.), Santorini, Greece, June 15-18.

Gaspar, B., Naess, A., Leira, B.J. \& Guedes Soares, C. (2014c). System Reliability Analysis by Monte Carlo Based Method and Finite Element Structural Models. Journal of Offshore Mechanics and Arctic Engineering 136(3), 031603-1-031603-9.

Gaspar, B., Teixeira, A.P., Guedes Soares, C. \& Wang, G. (2011). Assessment of IACS-CSR Implicit Safety Levels for Buckling Strength of Stiffened Panels for Double Hull Tankers. Marine Structures 24(4), 478-502.

Hurtado, J.E. \& Alvarez, D.A. (2001). Neuralnetwork-based Reliability Analysis: A Comparative Study. Computer Methods in Applied Mechanics and Engineering 191(1-2), 113-32.

Kaymaz, I. (2005). Application of Kriging Method to Structural Reliability Problems. Structural Safety 27(2), 133-51.
Matthies, H.G., Brenner, C.E., Bucher, C.G. \& Guedes Soares, C. (1997). Uncertainties in Probabilistic Numerical Analysis of Structures and Solids - Stochastic Finite Elements. Structural Safety 19(3), 283-336.

Olsson, A., Sandberg, G. \& Dahlblom, O. (2003). On Latin Hypercube Sampling for Structural Reliability Analysis. Structural Safety 25(1), 47-68.

Paik, J.K., Kim, B.J. \& Seo, J.K. (2008). Methods for Ultimate Limit State Assessment of Ships and Ship-shaped Offshore Structures: Part II Stiffened Panels. Ocean Engineering 35(2), 271-80.

Romero, V.J., Swiler, L.P. \& Giunta, A.A. (2004). Construction of Response Surfaces Based on Progressive-lattice-sampling Experimental Designs with Application to Uncertainty Propagation. Structural Safety 26(2), 201-19.

Santner, T.J., Williams, B.J. \& Notz, W.I. (2003). The Design and Analysis of Computer Experiments. Springer, New York.

Schöbi, R. \& Sudret, B. (2014). Combining Polynomial Chaos Expansions and Kriging for Solving Structural Reliability Problems. In Proceedings of the 7th International Conference on Computational Stochastic Mechanics (CSM-7), Deodatis, G. \& Spanos, P.D. (eds.), Santorini, Greece, June 15-18.

Stocki, R., Kolanek, K., Knabel, J. \& Tauzowski, P. (2009). FE Based Structural Reliability Analysis using STAND Environment. Computer Assisted Mechanics and Engineering Sciences 16, 35-58.

Sudret, B. (2012). Meta-models for Structural Reliability and Uncertainty Quantification. In Fifth Asian-Pacific Symposium on Structural Reliability and its Applications (5APSSRA), Phoon, K.K., Beer, M., Quek, S.T. \& Pang, S.D. (eds.), 53-76, Singapore.

Teixeira, A.P. \& Guedes Soares, C. (2007). Probabilistic Modelling of the Ultimate Strength of Plates with Random Fields of Corrosion. In Computational Stochastic Mechanics, Deodatis, G. \& Spanos, P.D. (eds.), 653-61, Millpress, Rotterdam.

Zou, T., Mahadevan, S., Mourelatos, Z. \& Meernik, P. (2002). Reliability Analysis of Automotive Body-door Subsystem. Reliability Engineering \& System Safety 78(3), 315-24. 